\title{
ABORDAGENS SOBRE O CONCEITO DE DERIVADA EM TESES E DISSERTAÇÕES DEFENDIDAS NO BRASIL
}

\author{
APPROACHES ABOUT THE CONCEPT OF DERIVATIVE IN THESES AND \\ DISSERTATIONS DEVELOPED IN BRAZIL
}

\section{ENFOQUES SOBRE EL CONCEPTO DE DERIVADOS EN TESIS Y DISERTACIONES DEFENDIDAS EN BRASIL}

\section{Maria Alice de Vasconcelos Feio Messias* (D) 8}

\begin{abstract}
RESUMO
Apresenta-se neste artigo um levantamento de teses e dissertações defendidas no Brasil que contemplaram o conceito de derivada como parte de seus objetos de estudo. Para tanto, efetivamos uma busca no Catálogo de Teses e Dissertação da Coordenação de Aperfeiçoamento de Pessoal de Nível Superior (CAPES). O termo "Conceito de Derivada" foi utilizado como chave de pesquisa, de maneira que consideramos os trabalhos vinculados às áreas de conhecimento de Ensino ou Ensino de Ciências e Matemáticas. Nessas condições, identificamos um total de 17 trabalhos, entre teses e dissertações, defendidos no período de 2008 a 2018. Evidenciamos que tais pesquisas foram realizadas no âmbito de Programas de Pós-Graduação em Educação Matemática, Ensino, Ensino de Ciências e Matemática e Ensino de Matemática. Em geral, o objeto de pesquisa desses trabalhos esteve vinculado ao ensino e aprendizagem de derivada, bem como às múltiplas compreensões de estudantes acerca desse conceito, como por exemplo, aquelas relacionadas à taxa de variação média e instantânea, coeficiente angular da reta tangente à curva, estudo de variabilidade, dentre outros aspectos.
\end{abstract}

Palavras-chave: Teses e Dissertações. Ensino. Ensino de Ciências e Matemáticas. Conceito de Derivada.

\begin{abstract}
We present in this article a survey of theses and dissertations developed in Brazil that contemplated the concept of derivative as part of its object study. Therefore, we carried out a search in the Catalog of Theses and Dissertations of the Coordination for the Improvement of Higher Education Personnel (CAPES). The term "Derivative Concept" was used as a research key; we considered the works linked to the areas of knowledge of Teaching or Teaching Science and Mathematics. Under such conditions, we identified a total of 17 papers, including theses and dissertations, defended from 2008 to 2018 . We noticed that such researches were carried out within the scope of Postgraduate Programs in Mathematical Education, Teaching, Science and Mathematics Teaching and Teaching of Mathematics. In general, the research object of these works was linked to the teaching and learning of derivative, as well as to the multiple understandings of students about this concept, such as those related to the mean and instantaneous rate of variation, angular coefficient of the tangent line to the curve, variability study, among other aspects.
\end{abstract}

Keywords: Theses and Dissertations. Teaching. Teaching of Science and Mathematics. Concept of Derivative.

\footnotetext{
* Doutorado em Educação em Ciências e Matemáticas pela Universidade Federal do Pará (UFPA). Professora do Departamento de Matemática, Estatística e Informática da Universidade do Estado do Pará (DMEI/UEPA), Professora da Universidade da Amazônia (UNAMA) e professora da Secretaria de Estado de Educação (SEDUC/PA), Belém, Pará, Brasil. Endereço para correspondência: Trav. dos Apinagés, 398, apto 401, Batista Campos, Belém, Pará, Brasil, CEP: 66033-170. E-mail: prof.alice.messias@ gmail.com
} 


\section{RESUMEN}

Este artículo presenta un relevamiento de tesis y disertaciones defendidas en Brasil que consideraron el concepto de derivada como parte de sus objetos de estudio. Por ello, realizamos una búsqueda en el Catálogo de Tesis y Disertaciones de la Coordinación de Perfeccionamiento del Personal de Educación Superior (CAPES). El término "Concepto Derivado" se utilizó como clave de investigación, por lo que consideramos los trabajos vinculados a las áreas de conocimiento de la Enseñanza o Enseñanza de las Ciencias y las Matemáticas. En estas condiciones, identificamos un total de 17 trabajos, entre tesis y disertaciones, defendidos en el período de 2008 a 2018. Se evidencia que dichas investigaciones se realizaron en el ámbito de los Programas de Posgrado en Educación, Docencia, Enseñanza de las Ciencias y Matemáticas. y Enseñanza de las matemáticas. En general, el objeto de investigación de estos trabajos estuvo vinculado a la enseñanza y aprendizaje de las derivadas, así como a las múltiples comprensiones de los estudiantes sobre este concepto, como los relacionados con la tasa de variación media e instantánea, coeficiente angular de la tangente a la curva, estudio de variabilidad, entre otros aspectos.

Palabras clave: Tesis y disertaciones. Enseñando. Enseñanza de las Ciencias y las Matemáticas. Concepto derivado.

\section{INTRODUÇÃO}

A apreensão de conceitos matemáticos se apresenta como um processo que não depende, simplesmente, da memorização de definições e/ou de habilidades operatórias específicas. Desse modo, entende-se que as ações interiorizadas por um indivíduo sobre determinado conceito e, principalmente, os significados dados a essas ações, são fundamentais para a compreensão de qualquer conhecimento matemático (MESSIAS, 2018).

Nessa perspectiva, admite-se que a aprendizagem matemática esteja condicionada a um amplo conjunto de interações inerentes à sua própria composição (BRANDEMBERG, 2010). Nesse sentido, admite-se que, para que um indivíduo compreenda um conceito, é preciso refletir sobre ele, e buscar nele (e em diferentes contextos) a abstração por meio de suas representações, generalizações e sintetizações.

Ressalta-se, ainda, que o fato de muitos indivíduos executarem tarefas sem refletir, ou mesmo entender os conceitos nelas envolvidos, prejudica a compreensão de ideias matemáticas e o consequente amadurecimento de construções mentais, que contribuem para a formação de um pensamento mais matematicamente maduro e coerente com a teoria formal.

Frente a tais constatações, e diante das experiências vivenciadas enquanto discente e docente em Cálculo, reitera-se a importância de efetivar estudos relativos à compreensão de estudantes acerca dos elementos que constituem essa área de conhecimento, de modo que as expressivas dificuldades inerentes ao entendimento sobre o conceito de Derivada, conforme apontado em diferentes estudos (PINTO, 2008; RAMOS, 2009; MATOS, 2013, IMAFUKU, 
2018, dentre outros), levaram à delimitação do campo de ação trabalho à problemática da aprendizagem desses conceitos.

O estudo da derivada é de fundamental importância para a construção de uma base mais sólida na esfera de conhecimentos do Cálculo. Logo, qualquer fragilidade no que tange ao entendimento desse conceito pode implicar em dificuldades na aprendizagem de outros conteúdos no âmbito do Cálculo, fato que reitera a importância dos aspectos apresentados nesse $\operatorname{artigo}^{1}$, o qual foi norteado pela seguinte questão: Como o conceito de derivada tem sido abordado em objetos de estudo de teses e dissertações defendidas no Brasil? A referida questão norteou a revisão de literatura efetivada nesse trabalho, cujo objetivo foi descrever como a aprendizagem do conceito de derivada tem sido contemplada enquanto objeto de estudo de pesquisas relativas às áreas de conhecimento de Ensino ou Ensino de Matemática no Brasil.

É importante ressaltar que o estudo descrito no decorrer desse estudo é de grande relevância, uma vez que possibilita ao leitor ter acesso a uma descrição prévia de teses e dissertações defendidas no Brasil, no período de 2008 a 2018, que contemplaram o conceito de derivada de uma função como parte de seus objetos de pesquisa, de maneira que seja possível que este trabalho sirva de subsídio para a realização de outras pesquisas vinculadas a esse tema. Ainda que outras revisões de literatura tenham sido desenvolvidas, destaca-se o fato de terem sido analisadas as diferentes formas que o referido conceito foi explorado nos trabalhos, independentemente da metodologia e/ou perspectiva teórica adotadas pelos pesquisadores.

Tendo como orientação a questão e objetivo previamente destacados, apresentam-se nos itens subsequentes aspectos concernentes à metodologia, bem como uma discussão acerca dos resultados obtidos.

\section{CONSIDERAÇÕES METODOLÓGICAS}

O estudo apresentado nesse trabalho se caracterizou como uma revisão de literatura, na qual tomou-se como fonte de dados pesquisas que contemplaram a aprendizagem do conceito de derivada como parte de seu objeto de pesquisa. As informações foram coletadas mediante os trabalhos indexados no Catálogo de Teses e Dissertações da CAPES². O termo "conceito de derivada" (entre aspas) foi definido como chave de pesquisa, de maneira que foram

\footnotetext{
${ }^{1} \mathrm{O}$ estudo apresentado nesse artigo apresenta os resultados de uma das etapas de uma pesquisa de iniciação científica desenvolvida pela autora no âmbito da Universidade da Amazônia (UNAMA/PA), tendo o apoio financeiro do Grupo SER Educacional.

${ }^{2}$ Disponível em http://catalogodeteses.capes.gov.br/catalogo-teses/\#!/ 
considerados os trabalhos relacionados às áreas de conhecimento de Ensino e Ensino de

Ciências e Matemáticas. Nessas condições, destacam-se os trabalhos elencados no quadro 1:

Quadro 1 - Teses/Dissertações defendidas no Brasil que contemplaram a aprendizagem do conceito de derivada como parte de seus objetos de estudo

\begin{tabular}{|c|c|c|c|c|}
\hline Programa/Instituição & Ano & Material & Autor(a) & Título \\
\hline \multirow{2}{*}{$\begin{array}{c}\text { Programa de Pós- } \\
\text { Graduação em Ensino de } \\
\text { Matemática } \\
\text { UFRJ }\end{array}$} & 2008 & Dissertação & $\begin{array}{l}\text { Gisela Maria } \\
\text { Da Fonseca } \\
\text { Pinto } \\
\end{array}$ & $\begin{array}{l}\text { Compreensão gráfica da derivada de } \\
\text { uma função real em um curso de } \\
\text { cálculo semipresencial }\end{array}$ \\
\hline & 2008 & Dissertação & $\begin{array}{l}\text { Selma Lopes } \\
\text { da Costa } \\
\text { André }\end{array}$ & $\begin{array}{c}\text { Uma Proposta para o Ensino do } \\
\text { Conceito de Derivada no Ensino Médio }\end{array}$ \\
\hline \multirow{2}{*}{$\begin{array}{l}\text { Programa de Estudos Pós- } \\
\text { Graduados em Educação } \\
\text { Matemática } \\
\text { PUC/SP }\end{array}$} & 2009 & Dissertação & $\begin{array}{l}\text { Vagner } \\
\text { Valeiros } \\
\text { Ramos }\end{array}$ & $\begin{array}{c}\text { Dificuldades e concepções de alunos de } \\
\text { um curso de Licenciatura em } \\
\text { Matemática, sobre derivada e suas } \\
\text { aplicações }\end{array}$ \\
\hline & 2012 & Dissertação & $\begin{array}{l}\text { Rogério dos } \\
\text { Santos Lobo }\end{array}$ & $\begin{array}{l}\text { O tratamento dado por livros didáticos } \\
\text { ao conceito de derivada }\end{array}$ \\
\hline \multirow{2}{*}{$\begin{array}{c}\text { Programa de Pós- } \\
\text { Graduação em Ensino de } \\
\text { Ciências e Matemática } \\
\text { Universidade Cruzeiro do } \\
\text { Sul (SP) }\end{array}$} & 2011 & Dissertação & $\begin{array}{c}\text { Andrea } \\
\text { Abdelmalack }\end{array}$ & $\begin{array}{c}\text { O ensino-aprendizagem-avaliação da } \\
\text { derivada para o curso de engenharia } \\
\text { através da resolução de problemas }\end{array}$ \\
\hline & 2016 & Tese & $\begin{array}{l}\text { Erica Marlucia } \\
\text { Leite Pagani }\end{array}$ & $\begin{array}{c}\text { O ensino-aprendizagem-avaliação de } \\
\text { derivadas no curso técnico integrado ao } \\
\text { Médio através da resolução de } \\
\text { problemas }\end{array}$ \\
\hline $\begin{array}{c}\text { Programa de Pós- } \\
\text { Graduação em Ensino de } \\
\text { Ciências e Matemática } \\
\text { PUC Minas }\end{array}$ & 2017 & Dissertação & $\begin{array}{l}\text { Allan Silva } \\
\text { Ferreira }\end{array}$ & $\begin{array}{c}\text { Diferentes abordagens do conceito de } \\
\text { derivada: uma proposta de investigação } \\
\text { matemática }\end{array}$ \\
\hline $\begin{array}{c}\text { Programa de Pós- } \\
\text { Graduação em Educação } \\
\text { Matemática } \\
\text { Universidade Estadual de } \\
\text { Santa Cruz } \\
\end{array}$ & 2015 & Dissertação & $\begin{array}{l}\text { Marcelo De } \\
\text { Araujo Lino }\end{array}$ & $\begin{array}{l}\text { Os registros de representação semiótica } \\
\text { na aprendizagem de derivada }\end{array}$ \\
\hline \multirow{2}{*}{$\begin{array}{c}\text { Programa de Pós- } \\
\text { Graduação em Educação } \\
\text { Matemática } \\
\text { Universidade Anhanguera } \\
\text { de São Paulo }\end{array}$} & 2014 & Dissertação & $\begin{array}{l}\text { Douglas Pães } \\
\text { Mação }\end{array}$ & $\begin{array}{l}\text { Uma proposta de ensino para o } \\
\text { conceito de derivada }\end{array}$ \\
\hline & 2018 & Tese & $\begin{array}{l}\text { Roberto Seidi } \\
\text { Imafuku }\end{array}$ & $\begin{array}{l}\text { O uso dos softwares SimCalc e } \\
\text { GeoGebra para enriquecimento da } \\
\text { imagem de conceito de derivada }\end{array}$ \\
\hline $\begin{array}{c}\text { Programa de Mestrado } \\
\text { Profissional em Educação } \\
\text { Matemática } \\
\text { Universidade Severino } \\
\text { Sombra }\end{array}$ & 2011 & Dissertação & $\begin{array}{l}\text { Monique } \\
\text { Sequeira } \\
\text { Lehmann }\end{array}$ & $\begin{array}{c}\text { Sequência didática para } \\
\text { conceitualização de derivada como taxa } \\
\text { de variação instantânea }\end{array}$ \\
\hline $\begin{array}{c}\text { Programa de Mestrado } \\
\text { Profissional em Educação } \\
\text { Matemática } \\
\text { UFOP }\end{array}$ & 2011 & Dissertação & $\begin{array}{l}\text { Daniel } \\
\text { Gustavo de } \\
\text { Oliveira }\end{array}$ & $\begin{array}{l}\text { Explorando o conceito de derivada em } \\
\text { sala de aula, a partir de suas aplicações } \\
\text { e sob uma perspectiva histórica }\end{array}$ \\
\hline $\begin{array}{c}\text { Programa de Pós- } \\
\text { Graduação em Ensino de } \\
\text { Ciências e Matemática } \\
\text { UEPB }\end{array}$ & 2012 & Dissertação & $\begin{array}{c}\text { Airlan } \\
\text { Arnaldo } \\
\text { Nascimento } \\
\text { De Lima }\end{array}$ & $\begin{array}{l}\text { Introduzindo o conceito de derivada a } \\
\text { partir da ideia de variação }\end{array}$ \\
\hline $\begin{array}{c}\text { Pós-Graduação em } \\
\text { Educação Matemática }\end{array}$ & 2013 & Dissertação & $\begin{array}{l}\text { Leozart da } \\
\text { Silva Matos }\end{array}$ & $\begin{array}{l}\text { Compreensões sobre derivada e integral } \\
\text { com o uso de um CAS on-line: um }\end{array}$ \\
\hline
\end{tabular}




\begin{tabular}{|c|c|c|c|}
\hline UFJF & & & $\begin{array}{c}\text { estudo com alunos do terceiro ano do } \\
\text { ensino médio }\end{array}$ \\
\hline
\end{tabular}

Fonte: Organizado pela autora.

Tendo em vista os critérios de busca estabelecidos, bem como as informações disponibilizadas no quadro 1 , observa-se que 2 teses e 12 dissertações $^{3}$, defendidas no período de 2008 e 2018, contemplaram o conceito de derivada como parte constituinte de seus objetos de estudo. Evidencia-se, nesse sentido, que a maioria dos trabalhos foram desenvolvidos no âmbito de instituições localizadas na região sudeste do Brasil (11 trabalhos), e os demais foram distribuídos entre as regiões sul e nordeste. A seção subsequente é dedicada à descrição das principais características desses trabalhos, os quais foram lidos na íntegra, sendo que tais características foram analisadas, especialmente, no que se refere às discussões relativas à aprendizagem do conceito de derivada.

\section{PESQUISAS \& APRENDIZAGEM DO CONCEITO DE DERIVADA: ALGUMAS CONSIDERAÇÕES}

Dentre os 14 trabalhos elencados no quadro 1, três não estão disponíveis para download na plataforma de Catálogo de Teses e Dissertações da CAPES, no site das instituições em que foram produzidas ou em formato impresso. São eles: Abdelmalack (2011), Pagani (2016) e Lino (2015). Portanto, esses trabalhos não são descritos ao longo dessa seção.

Observou-se, dentre os trabalhos produzidos, que a compreensão/concepção de estudantes universitários sobre derivada tem se configurado como objeto de pesquisa. Destacam-se, nesse sentido, as dissertações de Pinto (2008) e Ramos (2009).

Pinto (2008) desenvolveu uma pesquisa, cujo objetivo foi verificar de que forma estudantes de cursos de licenciatura em matemática, física e química, na modalidade de Educação a Distância, compreendem graficamente o conceito de derivada de uma função. Para tanto, solicitou que os estudantes resolvessem tarefas envolvendo o referido conceito, bem como participassem de entrevistas, relacionadas tanto aos hábitos de estudo dos sujeitos investigados quanto às respostas dadas para as questões solicitadas no decorrer das tarefas. As análises relativas à compreensão de estudantes sobre o conceito de derivada efetivadas à luz

\footnotetext{
${ }^{3}$ Reitera-se que os referidos trabalhos foram encontrados, tendo em vista o critério de busca estabelecido. Por isso, é compreensível que outras pesquisas que contemplem o conceito de derivada não tenham aparecido na busca realizada.
} 
dos estudos de Sfard (1991), Dubinsky (1991), Tall (1985, 1991,1994) e Asiala et al (1997). Em seus resultados, Pinto (2008) observou que a maioria dos estudantes apresenta dificuldades no que tange à interpretação geométrica de derivada, fato que os limita, por exemplo, a explorar esse objeto matemático do ponto de vista de práticas operatórias excessivas sem, no entanto, entender a natureza do referido conceito ou mesmo suas múltiplas representações e aplicações, seja no âmbito da matemática ou em outras áreas de conhecimento.

Em sua pesquisa, Ramos (2009) realizou um estudo diagnóstico, cujo objetivo foi investigar as principais dificuldades de estudantes universitários acerca do conceito de Derivada de uma função. Para tanto, os estudantes responderam a um conjunto de tarefas, as quais foram organizadas pelo autor que, por sua vez, norteou-se na Teoria de Registros e Representação Semiótica de Raymond Duval, fato que, dentre outros aspectos, influenciou na elaboração de atividades que exploravam representações algébricas e geométricas de função e derivada, bem como suas múltiplas relações entre si. Como resultados, Ramos (2009) observou, assim como Pinto (2008), que os estudantes costumam restringir-se às manipulações algébricas vinculadas a esse conhecimento, sem estender tais compreensões a sua representação geométrica ou a outros contextos (exclusivamente matemáticos ou não).

Verificou-se, em outra perspectiva, que o objeto "Derivada de uma Função" foi vinculado a pesquisas cujos sujeitos investigados eram estudantes de Ensino Médio. São eles: André (2008) e Matos (2013).

Em sua dissertação, André (2008) apresentou os resultados de uma pesquisa que teve como objetivo verificar a eficácia de uma proposta de ensino do conceito de derivada para estudantes do $2^{\circ}$ ano do Ensino Médio. As etapas contemplaram o estudo de variabilidade, taxa de variação média e instantânea e do conceito de derivada. A autora apontou para a importância de mudança na abordagem para ensinar determinados conceitos matemáticos, dentre eles o de derivada de uma função, destacando a relevância de partir do concreto para o abstrato no processo de ensino do referido conceito.

Matos (2013) desenvolveu uma pesquisa cujo objetivo foi investigar as compreensões de estudantes de $3^{\circ}$ ano do Ensino Médio, de uma escola particular, sobre os conceitos de derivada e integral em meio a um ambiente informatizado. Para tanto, o autor propôs uma série de atividades envolvendo derivada e integral a partir de uma abordagem mais conceitual (em detrimento de excessivas manipulações algébricas). No que tange ao conceito de derivada, Matos (2013) reforçou junto aos estudantes a ideia de taxa de variação e avaliou suas compreensões norteado pelos estudos sobre Imagem e Definição Conceitual de Tall e Vinner 
(1981) e Vinner (1991). Dentre os resultados obtidos, o autor observou que os estudantes apresentaram dificuldade no que se refere à ideia de taxa de variação instantânea. Ainda assim, tal abordagem levou-os a uma compreensão mais significativa do conceito de derivada, bem como de suas aplicações em diferentes contextos.

Uma das dissertações defendidas, Lobo (2012), teve como objeto de estudo a abordagem do conceito de derivada em livros didáticos. O objetivo do trabalho desenvolvido foi verificar como os livros didáticos abordam esse conteúdo. Para tanto, norteou-se nos apontamentos de Duval, no que se refere aos registros de representação semiótica, bem como nos princípios da análise de conteúdo de Bardin. Como resultados, o autor observou que muitos livros enfatizam a Derivada como taxa de variação, ainda que apenas um deles o tenha feito de maneira explícita. Poucos livros apresentavam a definição de função derivada, bem como demonstrações adjacentes a esse objeto matemático. A ideia de coeficiente angular também é comumente apresentada nos livros de Cálculo, ainda que, segundo a autora, a ênfase é dada às manipulações algébricas adjacentes a esse conceito em grande parte desses materiais.

Evidenciou-se que a maioria dos trabalhos defendidos apresentam pesquisas voltadas para a apresentação de propostas para ensinar o conceito de derivada para estudantes universitários da área de exatas, como é o caso dos cursos de engenharia e licenciatura em matemática, por exemplo. Destacam-se, nesse sentido, Lehman (2011), Oliveira (2011), Lima (2012), Mação (2014), Ferreira (2017) e Imafuku (2018).

Lehman (2011) apresentou, em sua dissertação, um estudo constituído de três estágios. No primeiro, a autora aplicou um teste diagnóstico sobre problemas envolvendo o conceito de derivada. Em seguida, destaca uma sequência de ensino desenvolvida em sua pesquisa, e norteada por situações-problemas, na qual foram enfatizados três temas: Construção, leitura e interpretação de gráficos; Variação de funções, Taxa de variação média; e Reta secante como taxa de variação média e Reta tangente como taxa de variação instantânea. Após participarem das aulas, nas quais foram desenvolvidos os temas contemplados na sequência de ensino elaborada pela autora, os sujeitos investigados responderam a um teste diagnóstico a posteriori, para que fosse possível verificar se os estudantes apreenderam os conhecimentos abordados na sequência e se haviam sido apreendidos de maneira adequada. A autora observou que os aspectos abordados nas atividades viabilizaram a compreensão dos sujeitos participantes da pesquisa.

Oliveira (2011) destacou em seu trabalho alguns métodos históricos relativos ao conceito de derivada sob a perspectiva do estudo da reta tangente a uma curva. Nesse sentido, 
o autor teve como objetivo viabilizar a compreensão do referido conceito a partir de alguns de seus elementos históricos. Para alcançar seu objetivo, ele desenvolveu uma sequência para ensinar um grupo de 38 alunos vinculados aos cursos de Matemática e Estatística de uma universidade pública localizada em Ouro Preto (MG). Dentre seus principais resultados, o autor observou que os sujeitos da pesquisa conseguiram apreender o conceito de derivada, fato que os permitiram reiterar a relevância de considerar a componente histórica para ensinar os diferentes objetos matemáticos, uma vez que tal proposta pode proporcionar uma aprendizagem mais significativa para os estudantes, além de motivá-los no decorrer do processo de construção do conhecimento.

A pesquisa desenvolvida por Lima (2012) teve o objetivo de elaborar, aplicar e analisar uma sequência didática elaborada para auxiliar a construção do conceito de derivada na perspectiva da noção de variação. Para tanto, realizou seu estudo com estudantes de Cálculo de uma universidade pública localizada no estado da Paraíba (Brasil), e teve como fundamentação teórica os apontamentos de Tall e Vinner (1981) e Vinner (1991) sobre Imagem e Definição Conceitual, bem como de Grattan-Guiness (1997). Em seus resultados, o autor observou que a maioria dos sujeitos participantes da pesquisa conseguiu construir de maneira adequada o conceito de derivada sob o ponto de vista de medida de variação, tendo, portanto, desenvolvido a partir da sequência elaborada, as noções de velocidade instantânea, taxa de variação e coeficiente angular da reta.

Mação (2014), em sua pesquisa, teve como objetivo apresentar abordagens para o ensino de derivada a partir de três perspectivas: a geométrica, como taxa e como aproximação. Segundo o autor, cada uma dessas perspectivas traz consigo características dos Três Mundos da Matemática de David Tall que, por sua vez, foi tomado como fundamentação teórica do estudo realizado. Em cada abordagem, os sujeitos da pesquisa foram colocados diante de situações problemas que os possibilitassem construir o referido conceito da maneira mais autônoma possível, ou seja, com o mínimo de intervenção do professor. Em seus resultados, o autor disponibilizou uma análise das situações à luz dos três mundos da matemática, fato que pode subsidiar a prática de professores de Cálculo e, consequentemente, a aprendizagem dos estudantes, tanto no que se refere ao conceito de derivada quanto de outros a ele adjacentes.

Em sua pesquisa, Ferreira (2017) objetivou elaborar, aplicar e analisar uma proposta de ensino para introduzir o conceito de derivada de uma função. Para tanto, norteou-se nos pressupostos da investigação matemática e do uso de Tecnologias de Informação e Comunicação, particularmente, do aplicativo Geogebra para smartphones. Nessa perspectiva, 
o autor elaborou um material de apoio para professores de Cálculo no qual o conceito de derivada é apresentado sob diferentes abordagens, dentre elas, a algébrica, a geométrica e, também, a partir de uma compreensão histórica da construção desse conceito.

Imafuku (2018) realizou um estudo cujo objetivo foi investigar a contribuição da utilização de softwares no ensino de derivada de maneira a viabilizar a compreensão de suas múltiplas representações, fato que, segundo o autor, pode enriquecer a imagem conceitual dos estudantes no âmbito do Cálculo. Para tanto, o autor elaborou, inicialmente, um questionário para os estudantes, com o intuito de verificar suas concepções acerca do referido conceito. Em seguida, realizou junto a esses sujeitos atividades no ambiente computacional, especificadamente por meio dos softwares Geogebra e SimCalc. Tais atividades apresentavam diferentes interpretações sobre derivada. Suas análises foram fundamentadas pelos estudos relativos aos três mundos da matemática, bem como pela teoria sobre imagem e conceitual. Dentre seus resultados, o autor observou que as múltiplas representações dinâmicas e síncronas dos referidos softwares foram fundamentais e facilitaram a realização de diferentes atividades, dentre elas, aquelas voltadas para a ideia de taxa de variação e aproximação, fato que permitiu a ampliação da imagem conceitual dos sujeitos sobre o conceito de derivada.

Tendo em vista o que fora apontado acerca das teses e dissertações defendidas nas áreas de Ensino e Ensino de Ciências e Matemáticas, que tiveram a aprendizagem do conceito de derivada como parte de seus objetos de estudo, apresenta-se a seguir o quadro 2, o qual, em síntese, destaca as principais características das pesquisas apresentadas nesses trabalhos.

Quadro 2 - Principais características das teses e dissertações disponíveis que contemplaram a aprendizagem do conceito de derivada como parte de seu objeto de estudo (2008-2018)

\begin{tabular}{|c|c|c|}
\hline Material & Objeto de Pesquisa & Total de trabalhos \\
\hline \multirow{3}{*}{ Dissertação } & Ensino/Compreensão de estudantes universitários sobre derivada & 7 \\
\hline & Ensino/Compreensão do Conceito de derivada no Ensino Médio & 2 \\
\hline & Conceito de derivada em livros didáticos & 1 \\
\hline \multirow{3}{*}{ Tese } & Ensino/Compreensão de estudantes universitários sobre derivada & 1 \\
\hline & Ensino/Compreensão do Conceito de derivada no Ensino Médio & - \\
\hline & Conceito de derivada em livros didáticos & - \\
\hline \multicolumn{2}{|r|}{ Total Geral } & 11 \\
\hline
\end{tabular}

Fonte: Organizado pela autora.

Verificou-se, a partir do quadro 2, que na maioria das pesquisas os autores optaram por investigar situações relativas ao ensino do conceito de derivada para estudantes de nível superior (total de seis trabalhos). Nesse sentido, observa-se que grande parte dos materiais 
encontrados e disponíveis para download, na ocasião em que o levantamento foi realizado, são dissertações de mestrado (total de 10 trabalhos).

Ressalta-se, mediante o quadro 2, que os trabalhos contemplados nessa revisão de literatura tiveram seus objetos de estudo relacionados a três categorias: (i) Ensino/Compreensão de estudantes universitários sobre o conceito de derivada, (ii) Ensino/Compreensão do conceito de derivada no ensino médio e (iii) Conceito de derivada em livros didáticos.

Observou-se, nesse sentido, que os trabalhos inseridos na categoria (i) apresentaram ou estudos diagnósticos acerca da compreensão dos estudantes sobre o referido conceito - e , nesse sentido, tiveram como quadro teórico vinculado a algumas teorias no âmbito do Pensamento Matemático Avançado (TALL; VINNER, 1981; DUBINSKY, 1991; dentre outros), ou desenvolveram atividades sobre derivada junto a discentes de cursos de Cálculo.

No que se refere à categoria (ii), verificou-se duas dissertações em que os autores desenvolveram o conceito de derivada por meio de atividades junto a estudantes do $3^{\circ}$ ano do nível médio. Nesse sentido, observou-se que eles fizeram o uso de uma abordagem vinculada, especialmente, à interpretação desse conceito como taxa de variação. Um dos trabalhos, o de Matos (2013), se apoiou nos estudos sobre imagem e definição conceitual de Tall e Vinner (1981) para averiguar a compreensão dos sujeitos que participaram da pesquisa.

Apenas um dos trabalhos destacados nesse levantamento teve o objeto de pesquisa inserido na categoria (iii). Nesse caso, autor norteou-se na teoria de análise de conteúdo de Bardin, e seus critérios estiveram relacionados com as diferentes abordagens/interpretações sobre derivada presentes nos materiais analisados.

Tendo em vista o que foi apresentado no decorrer do texto, destaca-se, no tópico a seguir, alguns apontamentos acerca da questão norteadora e objetivo delineados para esse trabalho.

\section{CONSIDERAÇÕES FINAIS}

"Como o conceito de derivada tem sido abordado em objetos de estudo em teses e dissertações defendidas no Brasil? ” Foi essa a questão que norteou a revisão de literatura nesse trabalho, com o qual objetivou-se descrever como a aprendizagem do conceito de derivada tem sido contemplada enquanto objeto de estudo de pesquisas relativas às áreas de conhecimento de Ensino ou Ensino de Matemática no Brasil.

Observou-se, nesse sentido, que as dissertações e teses defendidas no Brasil, na perspectiva dos critérios de busca estabelecidos, encontram-se em três categorias distintas: 
Ensino/Compreensão de estudantes universitários sobre o conceito de derivada, Ensino/Compreensão do conceito de derivada no ensino médio e Conceito de derivada em livros didáticos. Sendo que múltiplas interpretações de estudantes acerca desse conceito foram destacadas nos resultados das pesquisas.

É interessante destacar que, considerando os critérios de busca estabelecidos para essa revisão de literatura, observou-se que poucas pesquisas no âmbito das áreas de Ensino ou Ensino de Ciências e Matemáticas no Brasil tiveram o conceito de derivada como parte constituinte de seu objeto de estudo. Além disso, vale ressaltar que as teses e dissertações explicitadas no decorrer desse trabalho foram defendidas exclusivamente em programa de pósgraduação provenientes de instituições localizadas no eixo sul/sudeste do país, com exceção do trabalho de Lima (2002), o qual foi desenvolvido no âmbito do Programa de Pós-Graduação em Ensino de Ciências e Matemática da Universidade Estadual da Paraíba.

Considera-se, por fim, que o estudo apresentado nesse artigo é de grande relevância, uma vez que, a partir do levantamento e descrição dos estudos, admitimos possibilidades de despertar o interesse de pesquisadores em desenvolver pesquisas vinculadas à compreensão, ensino, aprendizagem, construção, dentre outros aspectos, relativos ao conceito de derivada de uma função ${ }^{4}$.

\section{REFERÊNCIAS}

ABDELMALACK, A. O ensino-aprendizagem-avaliação da derivada para o curso de engenharia através da resolução de problemas. 2011. 175f. Dissertação (Mestrado) Universidade Cruzeiro do Sul, Programa de Pós-Graduação em Ensino de Ciências e Matemática, São Paulo, 2011.

ANDRÉ, S. L. C. Uma proposta para o ensino do conceito de derivada no ensino médio. 2008. 241f. Dissertação (Mestrado) - Universidade Federal do Rio de Janeiro, Programa de Pós-Graduação em Ensino de Matemática, Rio de Janeiro, 2008.

BRANDEMBERG, J. C. Uma análise histórico-epistemológica do conceito de grupo. São Paulo: Livraria da Física, 2010.

FERREIRA, A. S. Diferentes abordagens do conceito de derivada: uma proposta de investigação matemática. 2017. 158f. Dissertação (Mestrado) - Pontifícia Universidade Católica de Minas, Programa de Pós-Graduação em Ensino de Ciências e Matemática, Belo Horizonte, 2017. Disponível em:

\footnotetext{
${ }^{4}$ Exemplifica-se, nesse sentido, a pesquisa em andamento desenvolvida pela autora desse artigo, cujo objetivo é analisar a compreensão de estudantes universitários no que tange ao conceito de derivada à luz dos apontamentos de Vinner (1991) sobre imagem e definição conceitual, enfatizando suas principais dificuldades. 
https://sucupira.capes.gov.br/sucupira/public/consultas/coleta/trabalhoConclusao/viewTrabalh oConclusao.jsf?popup $=$ true\&id trabalho=5503559 . Acesso em: 16/10/2020.

IMAFUKU, R. S. O uso dos softwares GeoGebra e SimCalc para enriquecimento da imagem de conceito de derivada. 2018. 437f. Tese (Doutorado) - Universidade Anhanguera de São Paulo, Programa de Pós-Graduação em Educação Matemática, São Paulo, 2018. Disponível em: https://sucupira.capes.gov.br/sucupira/public/consultas/coleta/trabalhoConclusao/viewTrabalh oConclusao.jsf?popup $=$ true\&id trabalho $=6367697$. Acesso em: 16/10/2020.

LIMA, A. A. N. Introduzindo o conceito de derivada a partir da ideia de variação. 2012. 11F. DISSERTAÇÃO (MESTRADO) - Universidade Estadual da Paraíba, Programa de PósGraduação em Ensino de Ciências e Matemática, Campina Grande, 2012. Disponível em: http://tede.bc.uepb.edu.br/jspui/bitstream/tede/1888/1/PDF\%20-

\%20Airlan\%20Arnaldo\%20Nascimento\%20de\%20Lima.pdf . Acesso em: 20/10/2020.

LINO, M. A. Os registros de representação semiótica na aprendizagem de derivada. 2015. Dissertação (Mestrado) - Universidade Estadual de Santa Cruz, Programa de PósGraduação em Educação Matemática, Ilhéus, 2015.

LOBO, R. S. O tratamento dado por livros didáticos ao conceito de derivada. 2012. 147f. Dissertação (Mestrado) - Pontifícia Universidade Católica de São Paulo, Programa de Estudos Pós-Graduados em Educação Matemática, São Paulo, 2012. Disponível em: https://tede2.pucsp.br/handle/handle/10939 . Acesso em: 18/10/2020.

MAÇÃO, D. P. Uma proposta de ensino para o conceito de derivada. 2014. 166F. Dissertação (Mestrado) - Universidade Anhanguera de São Paulo, Programa de PósGraduação em Educação Matemática, São Paulo, 2014. Disponível em: https://sucupira.capes.gov.br/sucupira/public/consultas/coleta/trabalhoConclusao/viewTrabalh oConclusao.jsf?popup $=$ true \&id trabalho=950152 . Acesso em: 16/10/2020.

MATOS, L. S. Compreensões sobre derivada e integral com o uso de um CAS on-line: um estudo com alunos do terceiro ano do ensino médio. 2013. 154f. Dissertação (Mestrado) - Universidade Federal de Juiz de Fora, Juiz de Fora, 2013. Disponível em: https://repositorio.ufjf.br/jspui/handle/ufjf/949 . Acesso em: 18/10/2020.

MESSIAS, M. A. V. F. Teorias cognitivas do Pensamento Matemático Avançado e a construção do conhecimento: um estudo envolvendo os conceitos de limite e continuidade. 2018. 188f. Tese (Doutorado em Educação em Ciências e Matemáticas) Instituto de Educação Matemática e Científica, Universidade Federal do Pará, Belém, 2018.

PAGANI, E. M. L. O ensino-aprendizagem-avaliação de derivadas no curso técnico integrado ao Médio através da resolução de problemas. 2016. 178f. Tese (Doutorado), Universidade Cruzeiro do Sul, Programa de Pós-Graduação em Ensino de Ciências e Matemática, São Paulo, 2016.

OLIVEIRA, D. G. Explorando o conceito de derivada em sala de aula, a partir de suas aplicações e sob uma perspectiva histórica. 2011. 78f. Dissertação (Mestrado) Universidade Federal de Ouro Preto, Programa de Pós-Graduação em Educação Matemática, 
Ouro Preto, 2011. Disponível em: https://www.repositorio.ufop.br/handle/123456789/2517 . Acesso em: 20/10/2020.

PINTO, G. M. F. Compreensão gráfica da derivada de uma função real em um curso de cálculo semipresencial. 2008. 108f. Dissertação (Mestrado) - Universidade Federal do Rio de Janeiro, Programa de Pós-Graduação em Ensino de Matemático, Rio de Janeiro, 2008.

RAMOS, V. V. Dificuldades e concepções de alunos de um curso de licenciatura em matemática sobre derivada e suas aplicações. 86f. 2009. Dissertação (Mestrado) Pontifícia Universidade Católica de São Paulo, Programa de Estudos Pós-Graduados em Educação Matemática, São Paulo, 2009. Disponível em:

https://tede2.pucsp.br/handle/handle/11380 . Acesso em: 20/10/2020.

LEHMANN, M. S. Sequência didática para conceitualização de derivada como taxa de variação instantânea. 2011. Dissertação (Mestrado) - Universidade Severino Sombra, Programa de Mestrado Profissional em Educação Matemática, Vassouras, 2011.

\title{
APÊNDICE 1
}

\section{AGRADECIMENTOS}

Ao Grupo Ser Educacional e Universidade da Amazônia (UNAMA).

\section{FINANCIAMENTO}

Não se aplica

\section{CONTRIBUIÇÕES DE AUTORIA}

Resumo/Abstract/Resumen: Maria Alice de Vasconcelos Feio Messias Introdução: Maria Alice de Vasconcelos Feio Messias

Referencial teórico: Maria Alice de Vasconcelos Feio Messias

Análise de dados: Maria Alice de Vasconcelos Feio Messias

Discussão dos resultados: Maria Alice de Vasconcelos Feio Messias

Conclusão e considerações finais: Maria Alice de Vasconcelos Feio Messias

Referências: Maria Alice de Vasconcelos Feio Messias

Revisão do manuscrito: Maria Alice de Vasconcelos Feio Messias

Aprovação da versão final publicada: Maria Alice de Vasconcelos Feio Messias

\section{CONFLITOS DE INTERESSE}

A autora declara não haver nenhum conflito de interesse de ordem pessoal, comercial, acadêmico, político e financeiro referente a este manuscrito.

DISPONIBILIDADE DE DADOS DE PESQUISA

A autora declara que disponibiliza todos os dados da pesquisa.

\section{CONSENTIMENTO DE USO DE IMAGEM}

Não se aplica.

\author{
APROVAÇÃO DE COMITÊ DE ÉTICA EM PESQUISA \\ Não se aplica.
}

\section{COMO CITAR - ABNT}

MESSIAS, Maria Alice de Vasconcelos Feio. Abordagens sobre o conceito de derivada em teses e dissertações defendidas no Brasil. REAMEC - Rede Amazônica de Educação em Ciências e Matemática. Cuiabá, v. 9, n. 2, $e 21042$, maio a agosto, 2021. https://doi.org/10.26571/reamec.v9i2.12160 


\section{COMO CITAR - APA}

Messias, M. A. de V. F. (2021 Abordagens sobre o conceito de derivada em teses e dissertações defendidas no Brasil. REAMEC - Rede Amazônica de Educação em Ciências e Matemática, 9(2), e21042. https://doi.org/10.26571/reamec.v9i2.12160

\section{LICENÇA DE USO}

Licenciado sob a Licença Creative Commons Attribution-NonCommercial 4.0 International (CC BY-NC 4.0). Esta licença permite compartilhar, copiar, redistribuir o manuscrito em qualquer meio ou formato. Além disso, permite adaptar, remixar, transformar e construir sobre o material, desde que seja atribuído o devido crédito de autoria e publicação inicial neste periódico.

\section{DIREITOS AUTORAIS}

Os direitos autorais são mantidos pelos autores, os quais concedem à Revista REAMEC - Rede Amazônica de Educação em Ciências e Matemática - os direitos exclusivos de primeira publicação. Os autores não serão remunerados pela publicação de trabalhos neste periódico. Os autores têm autorização para assumir contratos adicionais separadamente, para distribuição não exclusiva da versão do trabalho publicada neste periódico (ex.: publicar em repositório institucional, em site pessoal, publicar uma tradução, ou como capítulo de livro), com reconhecimento de autoria e publicação inicial neste periódico. Os editores da Revista têm o direito de proceder a ajustes textuais e de adequação às normas da publicação.

\section{PUBLISHER}

Universidade Federal de Mato Grosso. Programa de Pós-graduação em Educação em Ciências e Matemática (PPGECEM) da Rede Amazônica de Educação em Ciências e Matemática (REAMEC). Publicação no Portal de Periódicos UFMT. As ideias expressadas neste artigo são de responsabilidade de seus autores, não representando, necessariamente, a opinião dos editores ou da referida universidade.

\section{EDITOR}

Dailson Evangelista Costa (iD) 9

\section{HISTÓRICO}

Submetido: 09 de abril de 2021.

Aprovado: 25 de maio de 2021.

Publicado: 02 de agosto de 2021. 